\title{
Two-Dimensional Dynamics of Metal Nanoparticles on the Surface of Thin Polymer Films Studied with Coherent X Rays
}

\author{
S. Streit, ${ }^{1}$ C. Gutt,,${ }^{1 *}$ V. Chamard, ${ }^{2}$ A. Robert, ${ }^{3}$ M. Sprung, ${ }^{4}$ H. Sternemann, ${ }^{1}$ and M. Tolan ${ }^{1}$ \\ ${ }^{1}$ Fachbereich Physik, Universität Dortmund, Otto-Hahn-Strasse 4, 44221 Dortmund, Germany \\ ${ }^{2}$ TECSEN UMR CNRS 6122, Université Paul Cézanne, 13397 Marseille Cedex 20, France \\ ${ }^{3}$ European Synchrotron Radiation Facility, BP 220, 38043 Grenoble Cedex, France \\ ${ }^{4}$ Argonne National Laboratory, 9700 S. Cass Avenue, Bldg. 432 E003 Argonne, Illinois, 60439, USA
}

(Received 18 July 2006; published 24 January 2007)

\begin{abstract}
$\mathrm{X}$-ray photon-correlation spectroscopy is used to measure the dynamic structure factor $f(q, \tau)$ of gold particles moving on the surface of thin polymer films. Above the glass transition of the polymer the peculiar form $f(q, \tau) \sim \exp \left[-(\Gamma \tau)^{\alpha}\right]$ is found with $0.7<\alpha<1.5$, depending on sample age and temperature. The relaxation rates $\Gamma$ scale linearly with $q$, excluding a simple Brownian diffusive motion. This type of behavior, already observed in aging bulk soft matter systems, is explained by a power law distribution of particle velocities due to ballistic motion.
\end{abstract}

PACS numbers: 68.15.+e, 61.10.-i, 61.46.Df, 68.47.Mn

Composite systems of metallic nanoparticles dispersed in and on polymeric materials are of great interest from both technological and fundamental aspects, as their fine control and design lead to the fabrication of materials with novel electronic, magnetic, or catalytic properties [1]. These composite materials are often formed out of equilibrium inducing ordering and coalescence processes of the metal clusters $[2,3]$. As the technological properties of these materials depend on the structure and ordering of the metal nanoparticles a detailed understanding of the dynamic processes involved is desirable. Moreover, nonequilibrium systems often display dynamics which are more complex than, e.g., the simple Brownian diffusion. Currently there is considerable interest in nonequilibrium bulk soft matter systems like, e.g., clay suspensions [4], colloidal gels [5], or concentrated emulsions [6], which all show aging phenomena and a peculiar universal form of the dynamic structure factor. The 2D case, which often displays rich physical phenomena, is more difficult to study as it requires a surface sensitive technique able to probe the dynamics at the nanometer scale. In this context, $\mathrm{x}$-ray photon-correlation spectroscopy (XPCS) is a wellsuited technique $[7,8]$. The main advantage of using $x$ rays instead of direct methods of scanning probe microscopy is that averaged statistical information over the whole sample surface is obtained, which is essential for the analysis of aging effects. This work presents studies of the dynamic structure factor $f(q, \tau)$ with the relaxation time $\tau$ at wave vector transfers $q$ using grazing-incidence enhanced intensity XPCS at the maxima of the static scattering factor, thus probing for the first time 2D surface dynamics of colloids at nanometer length scales.

The metal/polymer sample is prepared by spincoating polystyrene from a toluene solution onto a polished silicon wafer without removing the native oxide layer. To extract residual toluene, the film is annealed at about $10^{-6} \mathrm{mbar}$ for $12 \mathrm{~h}$ at $30 \mathrm{~K}$ above the glass transition temperature of the bulk polymer $\left(T_{G}\right)$ and then cooled down to $300 \mathrm{~K}$ at a slow rate of about $20 \mathrm{~K} / \mathrm{h}$. The gold clusters are produced in a high vacuum chamber by thermal evaporation of gold from a molybdenum crucible onto the polymer surface with a deposition rate of about $0.5 \AA / \mathrm{min}$, while the polymer surface is held at room temperature, leading to the confinement of the clusters in a narrow surface region [9]. The metal-metal interaction being much stronger than the metal-polymer one, the gold atoms diffuse on the polymer surface during the evaporation until they nucleate or eventually desorb again [10]. As the surface tension of the metal is much larger than that of the polymer, for nominal gold layer thicknesses smaller than $10 \AA$ spherical, almost monodisperse clusters form [11]. The samples used for the experiments consist of an $800 \AA$ thick polystyrene film (molecular weight $34.4 \mathrm{~kg} / \mathrm{mol}, T_{G} \approx 353 \mathrm{~K}$ ) with a nominal gold "layer" thickness of $8 \AA$, which leads to a cluster coverage of about $32.5 \%$ of the polymer surface and an average cluster radius of about $18.5 \AA$. The static structure of similar systems has been studied by x-ray scattering [12] and transmission electron microscopy [13] methods.

The temperature evolution of the static structure of the cluster assembly has first been studied by incoherent grazing-incidence small angle scattering (GISAXS) [14] at the ID1 beam line (ESRF). The monochromatic x-ray beam (wavelength $\lambda=0.69 \AA$ ) impinges on the sample surface with an incidence angle $\alpha_{i}=0.15^{\circ}$, allowing for an enhancement of the near surface scattered intensity with regards to the bulk one. The diffuse intensity is mostly governed by the gold scattering, allowing for a precise monitoring of the cluster size and distribution. In the distorted wave Born approximation, the incoherently scattered intensity in the grazing-incidence geometry is proportional to $T\left(\alpha_{i}\right) P(q) f\left(q_{\|}\right) T\left(\alpha_{f}\right)$, where $T\left(\alpha_{i, f}\right)$ are the transmission functions for the incident and exit beams, $P(q)$ is the form factor of a single spherical particle (i.e., 
the squared modulus of the Fourier transform of a sphere whose size presents a Gaussian distribution) and $f\left(q_{\|}\right)$is the static structure factor, accounting for the lateral ordering of the clusters on the surface $\left(q_{\|}\right.$denoting the wave vector transfer parallel to the surface). It is given by [3]

$$
f\left(q_{\|}\right)=\frac{1-\exp \left(-2 q_{\|}^{2} \sigma_{d}^{2}\right)}{1-2 \exp \left(q_{\|}^{2} \sigma_{d}^{2}\right) \cos \left(q_{\|} d\right)+\exp \left(-2 q_{\|}^{2} \sigma_{d}^{2}\right)},
$$

where $d$ is the mean interparticle distance with a dispersion $\sigma_{d}$. Two $q_{\|}$scans taken at 300 and $450 \mathrm{~K}$ are presented on the inset of Fig. 1 together with their best fits.

Figure 1 shows the averaged lateral radius of the gold clusters of the sample as a function of temperature. A typical mean value of about $16.5 \pm 0.2 \AA$ is obtained at room temperature, in agreement with previous observations [12,13]. At $T=400 \mathrm{~K}$, a strong increase of the mean gold cluster radius is observed. The evolution of the integrated scattered intensity shows that the total number of scatterers remains constant in the near surface region. Thus the increase of the radius is related to the coalescence of the clusters. The driving force for this nonequilibrium dynamics is the attractive van der Waals interaction between the gold clusters, which ultimately leads to their coalescence. Together with the radius, the mean particle distance of about $77 \pm 2 \AA$ is extracted. The variations of the static structure factor with temperature are within the experimental uncertainties.

XPCS experiments have been performed in the same grazing-incidence geometry using the coherent beam of the ID10A beam line (ESRF) with $\lambda=1.16 \AA$, corresponding to a photon energy of $10.64 \mathrm{keV}$ in order to minimize beam damage. The energy bandpass of the single bounce $\mathrm{Si}(111)$

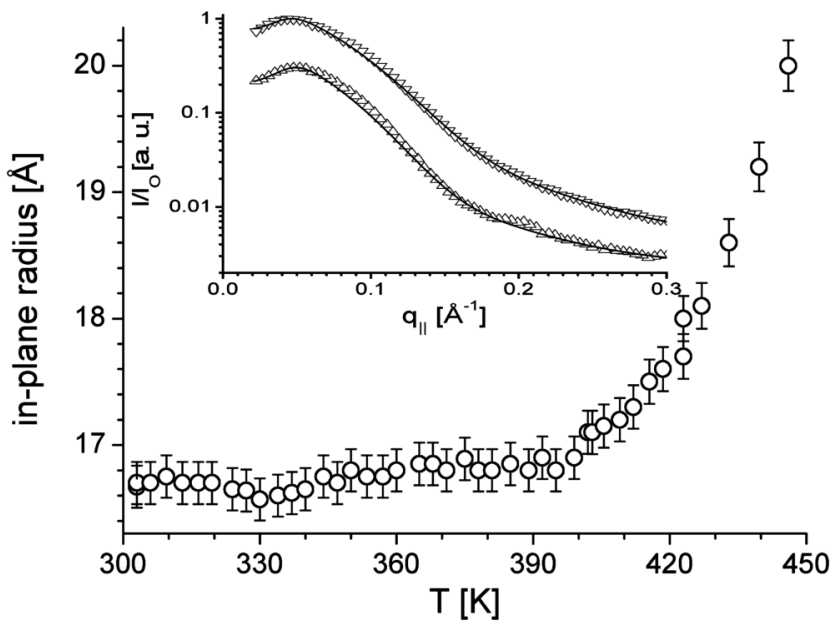

FIG. 1. Average in-plane radius of the gold clusters as a function of temperature as extracted from the incoherent GISAXS data. The inset presents two $q_{\|}$scans taken at 300 and $450 \mathrm{~K}$ (down and up triangles, respectively) together with their best fits. monochromator leads to a longitudinal coherence length $\xi=\lambda(\lambda / \Delta \lambda)=0.96 \mu \mathrm{m}$. A pinhole with a diameter of $20 \mu \mathrm{m}$ is placed upstream of the sample in order to obtain a collimated and transversely coherent beam. The beam is tilted vertically by a mirror onto the sample surface with $\alpha_{i}=0.15^{\circ}$. The measurements are performed around the region of total external reflection of the sample where the penetration depth of the evanescent wave is a few $\mathrm{nm}$, allowing for the surface sensitivity. A temperature range from $290 \mathrm{~K}$-well below $T_{G}$ - to $440 \mathrm{~K}$ is investigated, but due to the limited time window of the detector, decaying correlation functions can only be observed in the temperature interval between 400 and $440 \mathrm{~K}$. The effects of possible beam damage are further reduced by acquiring each data set at a new sample surface position. The scattered intensity is recorded by a direct illumination Princeton charge-coupled device (CCD) camera with $1242 \times 1152$ pixels (pixel size of $22.5 \times 22.5 \mu \mathrm{m}^{2}$ ). For each temperature, 400 images are taken with an exposure time of $10 \mathrm{~s}$ (plus readout time of about $2.5 \mathrm{~s}$ ). This kind of acquisition allows us to further monitor possible beam damages by controlling the average intensity of each picture. From the saved images the intensity autocorrelation function $g_{2}\left(q_{\|}, \tau\right)$ is calculated for each pixel. The final correlation function is obtained by averaging correlation functions with the same wave vector transfer, a so-called multiple-speckle analysis.

Figure 2 shows two images extracted from coherent GISAXS data measured at temperatures of 290 (left) and $420 \mathrm{~K}$ (right). Because of the use of a coherent x-ray beam, a speckle structure becomes visible, which reflects the exact positions and shapes of the cluster assembly. Interference between the polymer film and the silicon substrate leads to the well pronounced sheets of intensity oscillating along the $q_{z}$ direction. A broad maximum of the scattering factor is observed at $q_{\|}$values around $0.02 \AA^{-1}$, related to the lateral (nonperfect) order of the gold particles and the mean particle size. A comparison of the image measured from the "as prepared" sample at $290 \mathrm{~K}$ with the high temperature situation shows that the onset of the particle

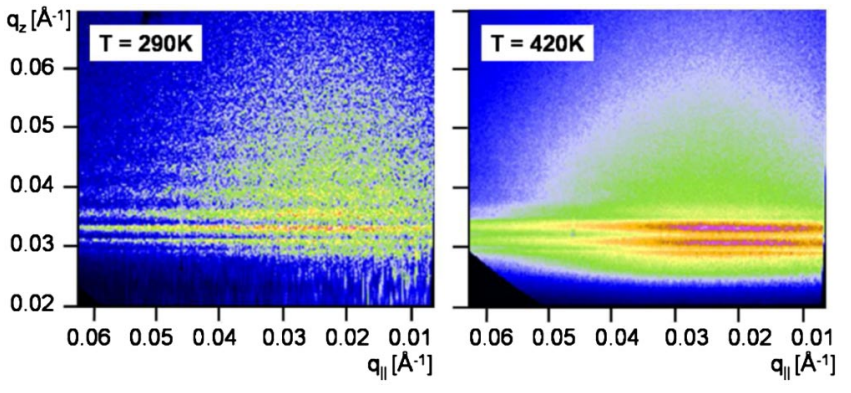

FIG. 2 (color online). CCD images of the coherent GISAXS patterns. The left picture was taken well below and the right above the glass transition, each one resulting from an accumulation of 400 frames of $10 \mathrm{~s}$ each. 
dynamics manifests itself by a smearing of the speckle pattern.

Details of the dynamic processes accompanied by the gold cluster coalescence can be revealed by calculating the intensity autocorrelation function from the images measured at different sample ages. For this purpose, each data set is divided into subsets of 100 images each covering a time window of about $20 \mathrm{~min}$. By use of the Siegert relation $g_{2}$ and the dynamic structure factor, $f\left(q_{\|}, \tau\right)$ are connected via $g_{2}\left(q_{\|}, \tau\right)=1+\beta\left(q_{\|}\right)\left|f\left(q_{\|}, \tau\right)\right|^{2} /\left|f\left(q_{\|}, 0\right)\right|^{2}$, which leads to the intensity autocorrelation function of

$$
g_{2}\left(q_{\|}, \tau\right)=1+\beta\left(q_{\|}\right) \exp \left[-2(\Gamma \tau)^{\alpha}\right],
$$

with $\beta\left(q_{\|}\right)$accounting for the $q$-dependent contrast in surface XPCS experiments [8], the relaxation rate $\Gamma$, and the exponent $\alpha$. The assumption of homodyne scattering for the use of the Siegert relation is valid because so far heterodyne scattering has been observed in surface scattering geometry only for very small in-plane wave vectors around $10^{-5} \AA^{-1}$ within the resolution element of the setup. The autocorrelation functions are shown in Fig. 3. The decay below 1 is due to the small time window and the average over pixels with similar $q_{\|}$values [15]. At room temperature the typical decay time is considerably larger than $20 \mathrm{~min}$, thus showing no decay of $g_{2}\left(q_{\|}, \tau\right)$, as the polymer matrix is still in a glassy state. Above the polymer glass transition the autocorrelation function decays faster

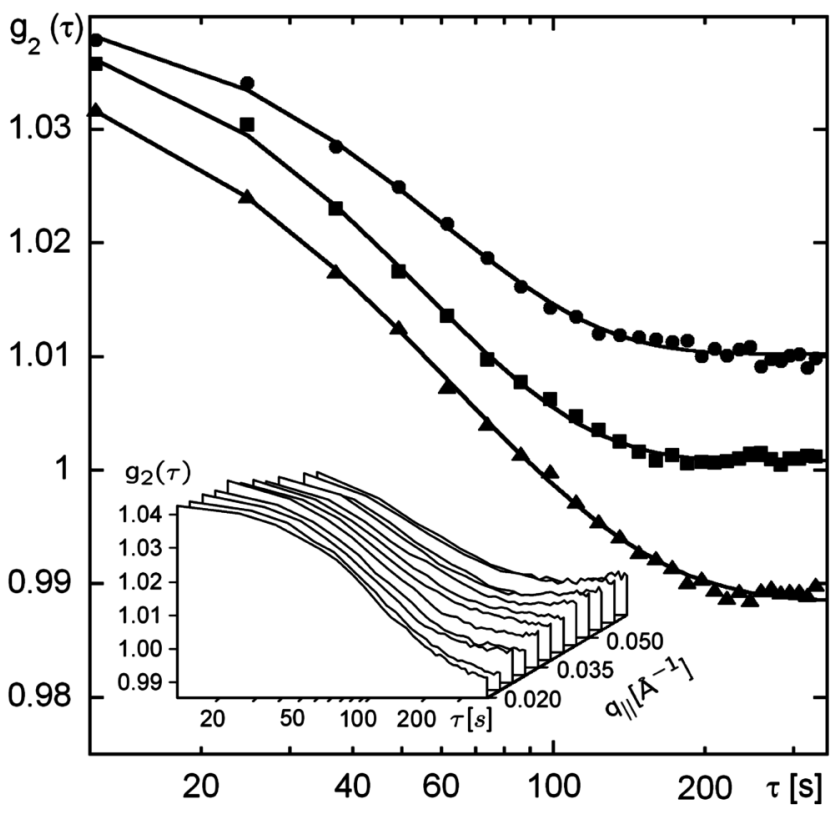

FIG. 3. Correlation functions $g_{2}(\tau)$ measured at 440 (circles), 420 (squares), and $400 \mathrm{~K}$ (triangles), respectively, for $q_{\|}=$ $0.051 \AA^{-1}$ and a sample age of 2462-3693 s. The curves are shifted vertically for reasons of clarity. The solid lines represent the fits according to Eq. (2). The inset shows the correlation functions for all measured wave vector transfers $q_{\|}$at $420 \mathrm{~K}$ for the same sample age. with increasing temperature. Thus the viscosity of the surrounding polymer matrix determines the mobility of the gold clusters.

The refinement of the data with Eq. (2) yields the following behavior of the exponent $\alpha$ as a function of sample age: at $400 \mathrm{~K}$ the exponent increases from a starting value of $0.7 \pm 0.1$ up to $1.0 \pm 0.1$, i.e., the form of the dynamic structure factor is evolving as a function of sample age from a stretched exponential at early times to a simple exponential behavior at later times. At $420 \mathrm{~K} \alpha$ increases from $0.7 \pm 0.2$ to $1.3 \pm 0.2$, corresponding to a transition from stretched to compressed exponential behavior. At $440 \mathrm{~K}$ the exponent has a nearly constant value of $1.5 \pm 0.2$ (see Table I). The relaxation rate $\Gamma\left(q_{\|}\right)$is found to vary linearly with $q_{\|}$, i.e., $\Gamma \sim q_{\|}$(Fig. 4) for all sample ages. Accompanied with the evolution of the exponent $\alpha$ as a function of sample age, the value of the relaxation rates $\Gamma$ also changes with age (see also Fig. 4). The characteristic relaxation time $t_{c}=\Gamma^{-1}$ is increasing with sample age; i.e., the system is slowing down with time, a behavior which has frequently been found in 3D colloidal suspensions showing hyperdiffusive dynamics. The gold cluster dynamics is clearly distinct from the simple Brownian motion. Because of the existence of aging and a stretched or compressed exponential and the fact that the data were taken at the maximum of the structure factor of the gold clusters where the scattering signal from the polymer surface is very low, capillary waves cannot explain the dynamics observed here. Cipelletti et al. proposed a model for this peculiar form of the dynamic structure factor [5] which is based on long ranging displacement fields induced by the particle motion. When two gold clusters are moving towards each other due to the attractive van der Waals forces they produce a strain field in the surrounding polymer matrix which causes other particles also to move. Such a type of motion is more ballistic than diffusive. Bouchaud and Pitard showed that the strain field model leads to the exponent $\alpha=\frac{3}{2}$ and the form $\Gamma \sim q_{\|}$[16] when the experimental time scale is considerably smaller than the typical coalescence time. This condition should be verified here, where the static structure factor does not show any change within a $10 \mathrm{~min}$ time scale. While these models are usually discussed in a $3 \mathrm{D}$ framework, our experiment clearly shows that the same dynamic structure factor is observed in 2D motion on a free surface. This type of ballistic motion can be described by a

TABLE I. Exponent $\alpha$ for different $T$ and sample ages. As $\alpha$ is independent of $q_{\|}$, the mean values of $\alpha$ over 15-20 $q_{\|}$values are given here together with the standard deviation.

\begin{tabular}{cccc}
\hline \hline Time [s] & $400 \mathrm{~K}$ & $420 \mathrm{~K}$ & $440 \mathrm{~K}$ \\
\hline $0-1231$ & $0.69 \pm 0.10$ & $0.72 \pm 0.16$ & $1.49 \pm 0.17$ \\
$1231-2462$ & $0.99 \pm 0.09$ & $1.00 \pm 0.15$ & $1.55 \pm 0.11$ \\
$2462-3693$ & $0.99 \pm 0.10$ & $1.33 \pm 0.15$ & $1.33 \pm 0.12$ \\
\hline \hline
\end{tabular}



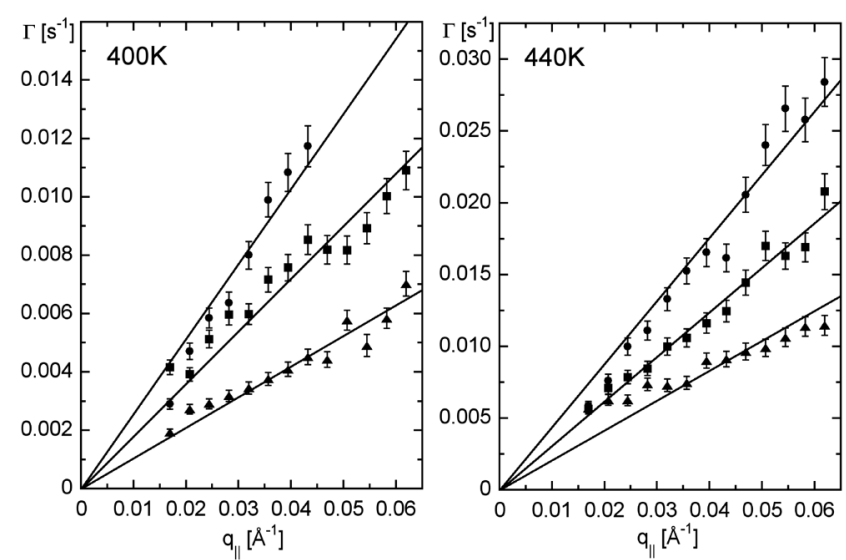

FIG. 4. Dispersion relations (relaxation rate $\Gamma$ vs $q_{\|}$) at 400 (left) and $440 \mathrm{~K}$ (right) presented for three different ages: 0-1231 s (circles), 1231-2462 s (squares), and 2462-3693 s (triangles). The solid lines are linear fits to the data.

power law velocity distribution of the particles which is determined by the derivative of the Levy stable law [17]. The mean particle velocity is $v_{0}=\Gamma / q_{\|}$and we find typical values of $v_{0}$ ranging from around $0.3 \AA / \mathrm{s}$ in the beginning to $0.1 \AA / \mathrm{s}$ at later times for $400 \mathrm{~K}$, and from around $0.5 \AA / \mathrm{s}$ at early times to $0.2 \AA / \mathrm{s}$ at the end for $440 \mathrm{~K}$. This shows that (i) the mean particle velocity increases with temperature, and (ii) due to aging, the particles slow down to around $40 \%-50 \%$ of their initial mean velocity. Within our observed time scale of around $10 \mathrm{~min}$, the gold clusters move over distances of $v_{0} t \approx$ $160 \AA$ in the first time region at $400 \mathrm{~K}$, then slow down and move $\approx 60 \AA$, whereas at $440 \mathrm{~K}$ they cover larger length scales of around $270 \AA$ in the beginning and $130 \AA$ in the end. As the distances covered by the clusters are larger than the mean cluster-cluster distance of about $80 \AA$ it is likely that the coalescence does not occur between first neighbors, but that the particle has to further move through the polymer fiber network.

In summary we measured the two-dimensional dynamics of gold clusters on thin polymer films on a nanometer length scale. The method of XPCS is uniquely suited for investigating these slow dynamics at large wave vector transfers. At temperatures above the glass transition of the surrounding polymer matrix the gold clusters start to move. This cluster dynamics is caused by the attractive van der Waals interaction between the clusters which ultimately leads to coalescence. The dynamic structure factor $f(q, \tau)$ of this dynamics has been measured, its peculiar form is reflecting a $2 \mathrm{D}$ hyper-diffusive or ballistic motion of the gold clusters. We observed aging phenomena as typical for out of equilibrium systems, and a transition from a stretched to a compressed exponential form of the dynamic structure factor.

This work was supported by the German Science Foundation (DFG project No. GU 535/2-1) and the Federal Ministry for Education and Research (BMBF project No. $05 \mathrm{KS} 4 \mathrm{PEA} / 3$ ). We thank H. T. Metzger for support at beam line ID1.

*Present address: Deutsches Elektronen Synchrotron (HASYLAB), Notkestrasse 85, 22607 Hamburg, Germany.

[1] W. A. Lopes and H. M. Jaeger, Nature (London) 414, 735 (2001).

[2] M. S. Kunz, K. R. Shull, and A. J. Kellock, J. Appl. Phys. 72, 4458 (1992).

[3] S. Narayanan, D. R. Lee, R. S. Guico, S. K. Sinha, and J. Wang, Phys. Rev. Lett. 94, 145504 (2005).

[4] R. Bandyopadhyay, D. Liang, H. Yardimci, D. A. Sessoms, M. A. Borthwick, S. G. J. Mochrie, J. L. Harden, and R. L. Leheny, Phys. Rev. Lett. 93, 228302 (2004).

[5] L. Cipelletti, S. Manley, R. C. Ball, and D. A. Weitz, Phys. Rev. Lett. 84, 2275 (2000).

[6] L. Cipelletti, L. Ramos, S. Manley, E. Pitard, D. A. Weitz, E. E. Pashkovski, and M. Johansson, Faraday Discuss. 123, 237 (2003).

[7] H. Kim, A. Rühm, L. B. Lurio, J. K. Basu, J. Lal, D. Lumma, S. G. J. Mochrie, and S. K. Sinha, Phys. Rev. Lett. 90, 068302 (2003).

[8] A. Madsen, T. Seydel, M. Tolan, and G. Grübel, J. Synchrotron Radiat. 12, 786 (2005).

[9] T. Strunskus, M. Kiene, R. Willecke, A. Thran, C. von Bechtolsheim, and F. Faupel, Mater. Corros. 49, 180 (1998).

[10] A. Thran, M. Kiene, V. Zaporojtchenko, and F. Faupel, Phys. Rev. Lett. 82, 1903 (1999).

[11] R. Weber, I. Grotkopp, J. Stettner, M. Tolan, and W. Press, Macromolecules 36, 9100 (2003).

[12] R. Weber, K.-M. Zimmermann, M. Tolan, J. Stettner, W. Press, O. H. Seeck, J. Erichsen, V. Zaporojtchenko, T. Strunskus, and F. Faupel, Phys. Rev. E 64, 061508 (2001).

[13] V. Zaporojtchenko, T. Strunskus, J. Erichsen, and F. Faupel, Macromolecules 34, 1125 (2001).

[14] H. Dosch, Critical Phenomena at Surfaces and Interfaces: Evanescent $X$-ray and Neutron Scattering, Springer Tracts in Modern Physics Vol. 126 (Springer, Berlin, 1992).

[15] D. Lumma, L. B. Lurio, S. G. J. Mochrie, and M. Sutton, Rev. Sci. Instrum. 71, 3274 (2000).

[16] J. P. Bouchaud and E. Pitard, Eur. Phys. J. E 6, 231 (2001).

[17] L. Ramos and L. Cipelletti, Phys. Rev. Lett. 87, 245503 (2001). 